\title{
Reduction in exacerbation of COPD in patients of advanced age using the Japanese Kampo medicine Dai-kenchu-to: a retrospective cohort study
}

This article was published in the following Dove Medical Press journal: International Journal of COPD

Taisuke Jo ${ }^{1,2}$

Nobuaki Michihata'

Hayato Yamana'

Yusuke Sasabuchi ${ }^{3}$

Hiroki Matsui ${ }^{4}$

Hirokazu Urushiyama

Akihisa Mitani²

Yasuhiro Yamauchi²

Kiyohide Fushimi ${ }^{5}$

Takahide Nagase ${ }^{2}$

Hideo Yasunaga ${ }^{4}$

'Department of Health Services Research, Graduate School of Medicine, The University of Tokyo,

Tokyo, Japan; ${ }^{2}$ Department of

Respiratory Medicine, Graduate

School of Medicine, The University

of Tokyo, Tokyo, Japan; ${ }^{3}$ Data Science

Center, Jichi Medical University,

Tochigi, Japan; ${ }^{4}$ Department of

Clinical Epidemiology and Health

Economics, School of Public Health,

The University of Tokyo, Tokyo,

Japan; ${ }^{5}$ Department of Health Policy and Informatics, Tokyo Medical and

Dental University Graduate School of Medicine, Tokyo, Japan
Correspondence: Taisuke Jo

Department of Respiratory Medicine,

Graduate School of Medicine,

The University of Tokyo, 7-3-I Hongo,

Bunkyo-ku, Tokyo I I 3-0033, Japan

$\mathrm{Tel}+8 \mid 3584$ I I I47

Fax $+8|3584| 1888$

Email jo-taisuke@umin.ac.jp
Purpose: Patients with symptomatic COPD are recommended to use inhaled bronchodilators containing long-acting muscarinic receptor antagonists (LAMAs). However, bronchodilators may cause gastrointestinal adverse effects due to anticholinergic reactions, especially in advanced-age patients with COPD. Dai-kenchu-to (TU-100, Da Jian Zhong Tang in Chinese) is the most frequently prescribed Japanese herbal Kampo medicine and is often prescribed to control abdominal bloating and constipation. The purpose of this study was to evaluate the role of Dai-kenchu-to as a supportive therapy in advanced-age patients with COPD.

Patients and methods: We used the Japanese Diagnosis Procedure Combination inpatient database and identified patients aged $\geq 75$ years who were hospitalized for COPD exacerbation. We then compared the risk of re-hospitalization for COPD exacerbation or death between patients with and without Dai-kenchu-to using 1-to-4 propensity score matching. A Cox proportional hazards model was used to compare the two groups. We performed subgroup analyses for patients with and without LAMA therapy.

Results: Patients treated with Dai-kenchu-to had a significantly lower risk of re-hospitalization or death after discharge; the HR was 0.82 (95\% CI, 0.67-0.99) in 1-to-4 propensity score matching. Subgroup analysis of LAMA users showed a significant difference in re-hospitalization or death, while subgroup analysis of LAMA non-users showed no significant difference.

Conclusion: Our findings indicate that Dai-kenchu-to may have improved the tolerability of LAMA in advanced-age patients with COPD and, therefore, reduced the risk of re-hospitalization or death from COPD exacerbation. Dai-kenchu-to may be recommended as a useful supportive therapy for advanced-age patients with COPD.

Keywords: TU-100, herbal medicine, muscarinic receptor antagonists, propensity score, survival analysis

\section{Introduction}

COPD is a common disease worldwide. Both COPD and aging lead to progressive deterioration in physical activity and lung function. Physical inactivity impacts the prognosis and can cause symptoms that may induce a vicious circle in advanced-age patients with COPD. ${ }^{1}$ In fact, functional constipation is one such symptom associated with physical inactivity, ${ }^{2}$ and patients with COPD who develop severe exacerbation requiring hospitalization are reportedly likely to be aged $\geq 75$ years. ${ }^{3}$

Patients with COPD are prone to acute worsening in their condition; this is known as COPD exacerbation. Because COPD is not curable, pharmacotherapy for COPD primarily aims to improve symptoms and prevent exacerbations. The use of long-acting bronchodilators such as long-acting muscarinic receptor antagonists (LAMAs) and 
long-acting $\beta 2$-agonists (LABAs) either alone or in combination is the first-line treatment for symptomatic COPD; such therapy is also effective for the prevention of COPD exacerbation. ${ }^{4,5}$ However, bronchodilators, particularly LAMAs, may potentially cause anticholinergic adverse events such as constipation in patients with COPD. ${ }^{6}$

Recent studies have focused on the contribution of the gastrointestinal tract in patients with COPD in both the stable state $^{7}$ and during exacerbation. ${ }^{8}$ Dai-kenchu-to (TU-100, Da Jian Zhong Tang in Chinese), the most frequently prescribed traditional Japanese herbal Kampo medicine, activates intestinal motility. ${ }^{9,10}$ Dai-kenchu-to consists of natural food ingredients: Zingiberis processum rhizome, Ginseng radix, Zanthoxyli piperiti pericarpium (Chinese Da Jian Zhong Tang contains Zanthoxyli pericarpium instead), and Saccharum granorum. ${ }^{11}$ Dai-kenchu-to has been widely prescribed in patients with gastrointestinal disorders such as postoperative paralytic ileus ${ }^{9,12-15}$ and chronic constipation ${ }^{16,17}$ and is referred to in the chronic constipation guideline in Japan. Dai-kenchuto is different from laxatives in that it is taken more regularly than laxatives, which are sometimes prescribed for single use or for a short term. Advanced-age patients with COPD constitutively experiencing abdominal bloating and/or chronic constipation are likely to be treated with Dai-kenchu-to. Bridging the concept of Oriental herbal medicine (ie, modulation of the respiratory system through the maintenance of digestive function) to modern medicine is desired.

The objective of this study was to evaluate the effect of Dai-kenchu-to on COPD exacerbation in patients of advanced age with the intention to provide evidence regarding supportive therapy for advanced-age patients with COPD.

\section{Patients and methods}

\section{Data source}

For this retrospective cohort study, we used the Japanese Diagnosis Procedure Combination (DPC) database. The DPC database with outpatient data includes discharge abstracts and administrative claims data from around 250 acute-care hospitals in Japan. The DPC database includes the following data: age, sex, body height and weight (body mass index), primary and comorbid diagnosis, Barthel index at admission and discharge, smoking history, A-DROP (Age-DehydrationRespiratory failure-Orientation disturbance-blood Pressure) score, Hugh-Jones score, information regarding operative procedures and anesthesia, discharge status, medications and treatment in both inpatient and outpatient settings, and dates of admission and discharge. All analyses and data reporting in this study complied with the Strengthening the Reporting of
Observational Studies in Epidemiology guideline. This study was approved by the Institutional Review Board of The University of Tokyo, which waived the requirement for informed patient consent because of the anonymous nature of the data.

\section{Patient selection}

We extracted inpatient data of patients aged $\geq 75$ years who were hospitalized for COPD exacerbation, and then extracted the outpatient data of these patients from July 2010 to March 2016. Patients with COPD were identified with the following ICD-10 codes: J41, J42, J43, J440, J441, and J449. Hospitalized patients with COPD exacerbation were identified with the following ICD-10 codes: J10, J11, J13, J14, J15, J16, J18, J20, J21, J22, J46, J170, J171, J178, J440, J441, J851, J12, A481, B012, B052, and B250. The Dai-kenchu-to group was defined as patients who received Dai-kenchu-to before or during hospitalization for COPD exacerbation. The control group comprised patients who did not receive Daikenchu-to throughout the observation period. Patients with no outpatient data before hospitalization, after hospitalization, or both before and after hospitalization and patients who died during the initial hospitalization for COPD exacerbation were excluded. Patients for whom Dai-kenchu-to was prescribed only after discharge from the initial hospitalization for COPD exacerbation were also excluded.

\section{Outcomes}

The outcome was re-hospitalization for COPD exacerbation or death.

\section{Statistical analyses}

We used the standardized difference to compare baseline characteristics, including comorbidities and treatments, between the Dai-kenchu-to group and the control group. Continuous variables are reported as mean \pm SD. Dichotomous parameters and categorical variables are reported as frequency. Missing data for categorical variables are categorized as missing. The baseline characteristics were sex, fiscal year, season at admission, residential region, Hugh-Jones dyspnea score at admission, activities of daily living scores at admission and discharge (Barthel index), age, body mass index, and frequency of COPD exacerbations before hospitalization. COPD exacerbation before hospitalization was defined by episodes in which corticosteroids and/or antibiotics and/or anti-flu medicines were prescribed (Table S1). Comorbidities (Table S2) and treatments assessed were lung cancer, other malignancy, interstitial pneumonia, bronchial asthma, bronchiectasis, bacterial lower respiratory tract infection, Mycobacterium 
infection, mycosis, cor pulmonale, congestive heart failure, ischemic heart disease, tachycardia, autoimmune disease, stroke, liver dysfunction, renal failure, gastroesophageal reflux disease, constipation and/or ileus, prostate hypertrophy, home ventilator support, home oxygen therapy, inhaled corticosteroids before hospitalization, long-acting muscarinic receptor antagonists before hospitalization, LABA before hospitalization, other medications for COPD before hospitalization, ambulance transport, corticosteroid therapy during hospitalization, mechanical ventilation during hospitalization, nasal tube feeding during hospitalization, surgery under general anesthesia during hospitalization, discharge to home, and length of stay. ICD-10 codes for comorbidities are shown in Table S2. An absolute standardized difference of $>10 \%$ was considered to indicate significant imbalance.

We performed propensity score analysis to account for the differences in baseline characteristics between the two groups. The propensity scores were estimated using a multivariable logistic regression model that included the aforementioned covariates as the dependent variables. ${ }^{18}$ We performed 1-to-4 matching between the Dai-kenchu-to group and the control group. Kaplan-Meier curves were drawn, and a log-rank test was then used to compare the survival curves of the two groups. A Cox proportional hazards model was used to estimate the HR of re-hospitalization for COPD exacerbation or death. Patients who were neither re-hospitalized for
COPD exacerbation nor died during the observation period were censored at the date of the last visit to each hospital.

All data were analyzed using SPSS version 23.0 (IBM Corporation, Armonk, NY, USA) and STATA software version 14.1 (StataCorp LP, College Station, TX, USA). Values of $P<0.05$ were considered to indicate statistical significance.

\section{Subgroup analyses}

We also performed subgroup analyses of patients with and without LAMA therapy. Patients with COPD who received either a LAMA alone or a LAMA in combination with a LABA (LABA/LAMA) before hospitalization may have been more likely to have worse symptoms and lower lung function with a higher risk of frailty and subsequent reduced gastrointestinal motility; therefore, we considered that they may benefit more from Dai-kenchu-to than patients without LAMA or LABA/LAMA therapy. Kaplan-Meier curves, a log-rank test, and a Cox proportional hazards model were used to compare the two groups.

\section{Results}

We identified 12,691 patients aged $\geq 75$ years who were hospitalized for COPD exacerbation and had outpatient data before and after hospitalization for COPD exacerbation. Among them, Dai-kenchu-to was prescribed to 518 patients before or during hospitalization for COPD exacerbation (Figure 1).

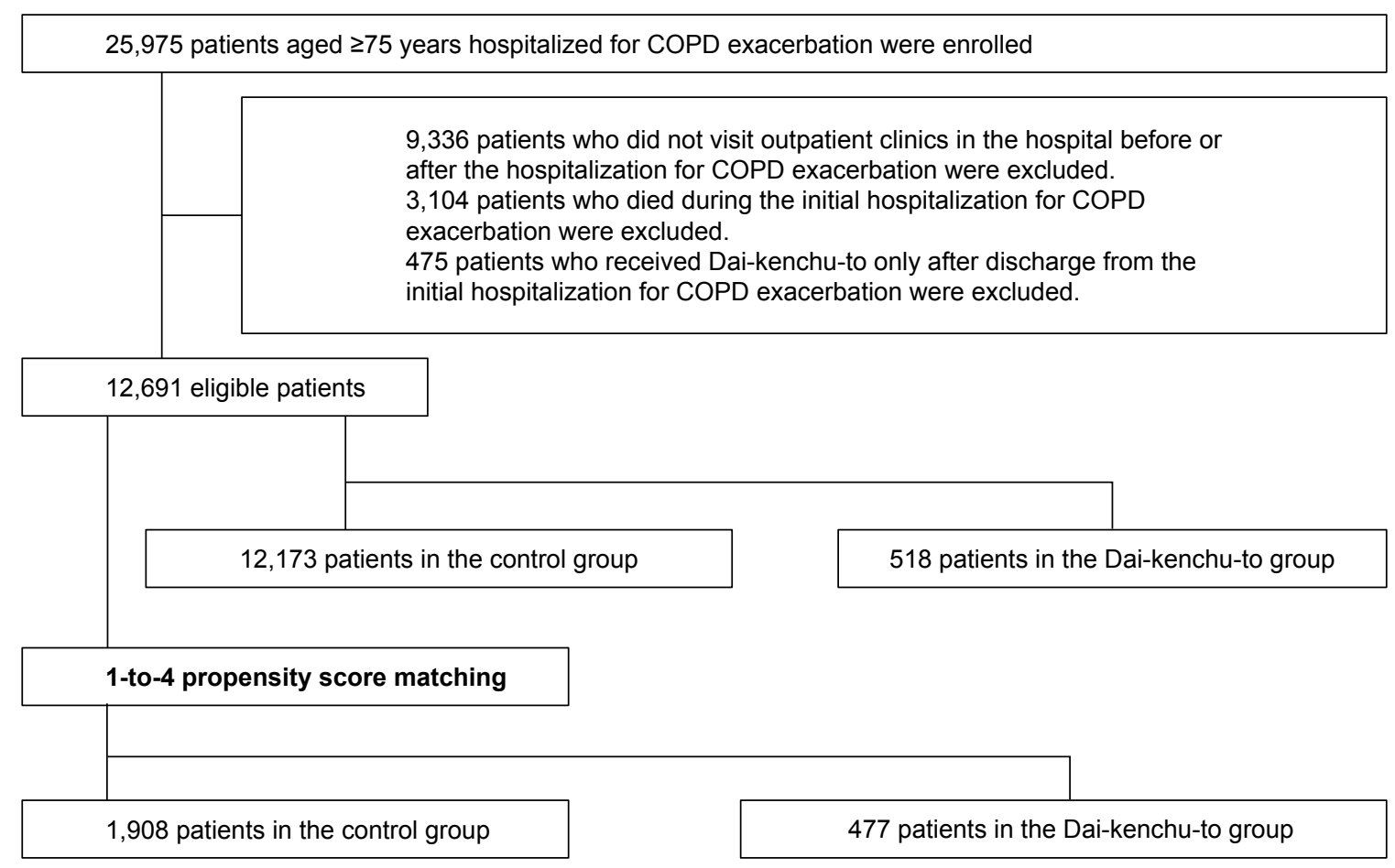

Figure I Flow diagram of patient selection. 
Table I Baseline characteristics of patients with COPD aged $\geq 75$ years with and without Dai-kenchu-to, before and after I-to- 4 propensity score matching

\begin{tabular}{|c|c|c|c|c|c|c|c|c|c|c|}
\hline \multirow[t]{3}{*}{ Characteristic (categorical) } & \multicolumn{5}{|c|}{ All patients } & \multicolumn{5}{|c|}{ After I-to-4 matching } \\
\hline & \multicolumn{2}{|c|}{$\begin{array}{l}\text { Control } \\
(n=\mid 2,173)\end{array}$} & \multicolumn{2}{|c|}{$\begin{array}{l}\text { Dai-kenchu-to } \\
(n=5 \mid 8)\end{array}$} & \multirow[t]{2}{*}{ s.d. } & \multicolumn{2}{|c|}{$\begin{array}{l}\text { Control } \\
(n=I, 908)\end{array}$} & \multicolumn{2}{|c|}{$\begin{array}{l}\text { Dai-kenchu-to } \\
(n=477)\end{array}$} & \multirow[t]{2}{*}{ s.d. } \\
\hline & \multicolumn{2}{|l|}{$\%$} & \multicolumn{2}{|l|}{$\%$} & & \multicolumn{2}{|l|}{$\%$} & \multicolumn{2}{|l|}{$\%$} & \\
\hline Sex (male) & \multicolumn{2}{|l|}{80.1} & \multicolumn{2}{|l|}{85.9} & -0.134 & \multicolumn{2}{|l|}{86.6} & \multicolumn{2}{|l|}{85.5} & 0.032 \\
\hline \multicolumn{11}{|l|}{ Fiscal year } \\
\hline 2010 & \multicolumn{2}{|l|}{12.3} & \multicolumn{2}{|l|}{8.7} & -0.125 & \multicolumn{2}{|l|}{7.4} & \multicolumn{2}{|l|}{8.2} & 0.027 \\
\hline 2011 & \multicolumn{2}{|l|}{16.6} & 9.9 & & -0.213 & 10.7 & & 9.2 & & $-0.05 I$ \\
\hline 2012 & 17.8 & & 16.4 & & -0.031 & 16.3 & & 16.6 & & 0.007 \\
\hline 2013 & 17.9 & & 17.8 & & 0.005 & 16.0 & & 18.2 & & 0.058 \\
\hline 2014 & 19.4 & & 24.7 & & 0.108 & 25.1 & & 24.1 & & -0.023 \\
\hline 2015 & 16.0 & & 22.6 & & 0.185 & 24.4 & & 23.7 & & -0.016 \\
\hline Seasons at admission & & & & & & & & & & \\
\hline Spring & 24.4 & & 25.7 & & 0.034 & 24.7 & & 25.8 & & 0.035 \\
\hline Summer & 23.0 & & 22.8 & & -0.016 & 24.0 & & 22.2 & & -0.042 \\
\hline Autumn & 23.3 & & 22.4 & & -0.031 & 24.2 & & 22.0 & & -0.052 \\
\hline Winter & 29.3 & & 29.2 & & 0.010 & 27.5 & & 30.0 & & 0.054 \\
\hline Residential regions & & & & & & & & & & \\
\hline Hokkaido and Tohoku & 9.0 & & 8.5 & & -0.009 & 13.1 & & 8.8 & & -0.136 \\
\hline Kanto & 28.7 & & 37.5 & & 0.197 & 31.5 & & 36.9 & & 0.114 \\
\hline Chubu & 20.3 & & 18.9 & & -0.022 & 20.6 & & 19.7 & & -0.022 \\
\hline Kansai & 14.8 & & 14.5 & & -0.056 & 14.3 & & 12.8 & & -0.043 \\
\hline $\begin{array}{l}\text { Chugoku, Shikoku, Kyushu, } \\
\text { and Okinawa }\end{array}$ & 27.3 & & 20.7 & & -0.142 & 20.6 & & 21.8 & & 0.029 \\
\hline $\begin{array}{l}\text { Hugh-Jones dyspnea score at } \\
\text { admission }\end{array}$ & & & & & & & & & & \\
\hline 1 & 10.0 & & 12.2 & & $0.08 I$ & 9.5 & & 13.0 & & 0.111 \\
\hline $2-3$ & 31.5 & & 26.5 & & -0.111 & 31.3 & & 27.0 & & -0.095 \\
\hline $4-5$ & 46.9 & & 46.9 & & 0.008 & 46.7 & & 46.8 & & 0.002 \\
\hline Missing & 11.7 & & 14.5 & & 0.063 & 12.5 & & 13.2 & & 0.020 \\
\hline ADL at admission (Barthel index) & & & & & & & & & & \\
\hline $95-100$ & 26.5 & & 30.5 & & 0.067 & 25.7 & & 28.5 & & 0.064 \\
\hline $55-90$ & 21.6 & & 18.5 & & -0.078 & 21.8 & & 19.1 & & -0.066 \\
\hline $0-50$ & 35.0 & & 35.1 & & 0.006 & 35.9 & & 36.5 & & 0.012 \\
\hline Missing & 16.9 & & 15.8 & & -0.003 & 16.7 & & 15.9 & & -0.020 \\
\hline ADL at discharge (Barthel index) & & & & & & & & & & \\
\hline $95-100$ & 13.8 & & 17.8 & & 0.078 & 11.7 & & 15.7 & & 0.118 \\
\hline $55-90$ & 20.4 & & 18.9 & & -0.023 & 19.9 & & 19.5 & & -0.009 \\
\hline $0-50$ & 55.7 & & 51.5 & & -0.080 & 57.3 & & 53.3 & & -0.082 \\
\hline Missing & 10.0 & & 11.8 & & $0.07 \mid$ & II.I & & 11.5 & & 0.013 \\
\hline Characteristic (numerical) & Mean & SD & Mean & SD & s.d. & Mean & SD & Mean & SD & s.d. \\
\hline Age (years) & 82.0 & 4.8 & 82.2 & 4.9 & 0.025 & 82.1 & 4.8 & 82.1 & 4.8 & -0.000 \\
\hline Body mass index $\left(\mathrm{kg} / \mathrm{m}^{2}\right)$ & 20.6 & 3.7 & 20.2 & 3.8 & -0.130 & 20.2 & 3.6 & 20.2 & 3.7 & -0.019 \\
\hline $\begin{array}{l}\text { Frequency of COPD exacerbation } \\
\text { before admission }\end{array}$ & 5.4 & 12.1 & 7.9 & 13.3 & 0.218 & 8.1 & 15.5 & 8.3 & 13.7 & -0.002 \\
\hline
\end{tabular}

Abbreviations: $A D L$, activities of daily living; s.d., standardized difference.

Table 1 shows the baseline characteristics in the Daikenchu-to group and the control group. The number of patients in the Dai-kenchu-to group and control group after 1-to-4 propensity score matching was 477 and 1,908, respectively. Comorbidities during the initial hospitalization and treatments for COPD before and during hospitalization are shown in Table 2. Malignancies other than lung cancer and constipation and/or ileus were more common in the Dai-kenchu-to group than the control group. Medications for COPD, including LAMAs, were more frequently administered in the 
Table 2 Comorbidities during hospitalization and treatments for COPD in patients aged $\geq 75$ years with and without Dai-kenchu-to

\begin{tabular}{|c|c|c|c|c|c|c|c|c|c|c|}
\hline \multirow[t]{3}{*}{ Comorbidities } & \multicolumn{5}{|c|}{ All patients } & \multicolumn{5}{|c|}{ After I-to-4 matching } \\
\hline & \multicolumn{2}{|c|}{$\begin{array}{l}\text { Control } \\
(n=12,173)\end{array}$} & \multicolumn{2}{|c|}{$\begin{array}{l}\text { Dai-kenchu-to } \\
(n=5 \mid 8)\end{array}$} & \multirow[t]{2}{*}{ s.d. } & \multicolumn{2}{|c|}{$\begin{array}{l}\text { Control } \\
(n=1,908)\end{array}$} & \multicolumn{2}{|c|}{$\begin{array}{l}\text { Dai-kenchu-to } \\
(\mathrm{n}=477)\end{array}$} & \multirow[t]{2}{*}{ s.d. } \\
\hline & \multicolumn{2}{|l|}{$\%$} & \multicolumn{2}{|l|}{$\%$} & & \multicolumn{2}{|l|}{$\%$} & \multicolumn{2}{|l|}{$\%$} & \\
\hline Lung cancer & \multicolumn{2}{|l|}{8.2} & \multicolumn{2}{|l|}{9.7} & 0.055 & \multicolumn{2}{|l|}{11.0} & \multicolumn{2}{|l|}{10.1} & -0.029 \\
\hline Other malignancy & \multicolumn{2}{|l|}{9.1} & \multicolumn{2}{|l|}{22.2} & 0.370 & \multicolumn{2}{|l|}{22.5} & \multicolumn{2}{|l|}{22.9} & 0.009 \\
\hline Interstitial pneumonia & \multicolumn{2}{|l|}{5.0} & \multicolumn{2}{|l|}{4.8} & -0.001 & \multicolumn{2}{|l|}{5.2} & \multicolumn{2}{|l|}{5.0} & -0.009 \\
\hline Bronchial asthma & 25.5 & & 25.3 & & -0.000 & 27.3 & & 25.6 & & -0.038 \\
\hline Bronchiectasis & 23.9 & & 24.3 & & 0.013 & 25.0 & & 24.3 & & -0.016 \\
\hline $\begin{array}{l}\text { Bacterial lower respiratory } \\
\text { tract infection }\end{array}$ & 85.4 & & 88.0 & & 0.080 & 89.0 & & 88.1 & & -0.030 \\
\hline Mycobacterium infection & 2.0 & & 1.2 & & -0.076 & 0.6 & & 1.1 & & 0.046 \\
\hline Mycotic infection & 1.4 & & 1.5 & & 0.038 & 1.8 & & 1.9 & & 0.008 \\
\hline Cor pulmonale & 1.0 & & 1.5 & & 0.047 & 1.6 & & 1.5 & & -0.013 \\
\hline Congestive heart failure & 21.3 & & 24.5 & & 0.093 & 25.9 & & 25.0 & & -0.023 \\
\hline Ischemic heart disease & 11.8 & & 11.6 & & 0.008 & 11.9 & & 12.4 & & -0.002 \\
\hline Tachycardia & 8.6 & & 8.9 & & 0.017 & 9.3 & & 9.2 & & -0.002 \\
\hline Autoimmune disease & 3.4 & & 2.7 & & -0.030 & 3.3 & & 2.9 & & -0.021 \\
\hline Stroke & 2.6 & & 1.9 & & -0.054 & 1.6 & & 1.7 & & 0.008 \\
\hline Liver dysfunction & 1.7 & & 1.4 & & -0.040 & 1.4 & & 1.3 & & -0.009 \\
\hline Renal failure & 4.7 & & 4.4 & & 0.003 & 4.8 & & 4.8 & & -0.000 \\
\hline GERD & 14.2 & & 16.2 & & 0.066 & 16.9 & & 16.6 & & -0.010 \\
\hline Constipation or ileus & 11.2 & & 18.9 & & 0.203 & 17.6 & & 18.5 & & 0.022 \\
\hline Prostate hypertrophy & 8.7 & & $1 \mathrm{I} .4$ & & 0.100 & 12.3 & & 11.7 & & -0.018 \\
\hline Treatments & $\%$ & & $\%$ & & s.d. & $\%$ & & $\%$ & & s.d. \\
\hline Home ventilatory support & 1.2 & & 1.2 & & 0.009 & 1.4 & & 1.3 & & -0.009 \\
\hline Home oxygen therapy & 14.9 & & 18.7 & & 0.100 & 17.3 & & 18.7 & & 0.035 \\
\hline ICS before admission & 27.6 & & 34.6 & & 0.148 & 34.8 & & 34.8 & & 0.001 \\
\hline LAMA before admission & 30.1 & & 39.2 & & 0.181 & 37.9 & & 39.0 & & 0.023 \\
\hline LABA before admission & 39.5 & & 50.0 & & 0.218 & 48.9 & & 50.5 & & 0.032 \\
\hline $\begin{array}{l}\text { Other medications for COPD } \\
\text { before admission }\end{array}$ & 58.8 & & 70.7 & & 0.250 & 71.5 & & 70.9 & & -0.014 \\
\hline Ambulance transport & 25.1 & & 25.1 & & -0.010 & 24.0 & & 23.9 & & -0.001 \\
\hline $\begin{array}{l}\text { Corticosteroids during } \\
\text { hospitalization }\end{array}$ & 35.9 & & 32.4 & & -0.075 & 32.7 & & 32.5 & & -0.004 \\
\hline $\begin{array}{l}\text { Mechanical ventilation during } \\
\text { hospitalization }\end{array}$ & 4.6 & & 5.0 & & -0.004 & 4.8 & & 4.4 & & 0.018 \\
\hline $\begin{array}{l}\text { Nasal tube feeding during } \\
\text { hospitalization }\end{array}$ & 1.8 & & 3.3 & & 0.095 & 2.6 & & 3.1 & & 0.031 \\
\hline $\begin{array}{l}\text { Surgery under general } \\
\text { anesthesia during } \\
\text { hospitalization }\end{array}$ & 0.5 & & 0.8 & & 0.041 & 0.8 & & 0.8 & & -0.000 \\
\hline Discharge to home & 91.7 & & 92.3 & & 0.046 & 94.0 & & 93.3 & & -0.030 \\
\hline Treatment (numerical) & Mean & SD & Mean & SD & s.d. & Mean & SD & Mean & SD & s.d. \\
\hline Length of stay & 18.9 & 17.5 & 19.6 & 19.7 & 0.020 & 19.3 & 17.1 & 19.2 & 19.3 & -0.003 \\
\hline
\end{tabular}

Abbreviations: GERD, gastroesophageal reflux disease; ICS, inhaled corticosteroids; LABA, long-acting $\beta$-agonist; LAMA, long-acting muscarinic receptor antagonist; s.d., standardized difference.

Dai-kenchu-to group. After 1-to-4 propensity score matching, the distributions of most covariates were well balanced between the two groups.

The median observation period after discharge from the first hospitalization for COPD exacerbation was 303 days
(IQR, 104-594 days) in the Dai-kenchu-to group and 308 days (IQR, 100-678 days) in the control group. During the observation period, $127(24.5 \%)$ and 3,379 (27.8\%) patients were either re-hospitalized for COPD exacerbation or died in the Dai-kenchu-to group and control group, respectively. 
Propensity scores were calculated using all the variables listed in Tables 1 and 2. After propensity score matching, the numbers of patients who were either re-hospitalized for COPD exacerbation or died during the observational period were $116(24.3 \%)$ and $536(28.2 \%)$ in the Dai-kenchu-to group and the control group, respectively.

Figure 2 and Table 3 show the results of the survival analyses after 1-to-4 propensity score matching. The log-rank test showed a statistically significant difference $(P=0.050)$ with better survival in the Dai-kenchu-to group (Figure 2A). In the analyses using the Cox proportional hazard model, the Dai-kenchu-to group showed a significantly reduced risk of re-hospitalization or death in the 1-to-4 propensity score analysis (HR, $0.82 ; 95 \% \mathrm{CI}, 0.67-0.99 ; P=0.050)$, as shown in Table 3.

In the subgroup analysis of patients with LAMA therapy, a significant difference in re-hospitalization or death was observed between the two groups (Figure 2B). Among patients without LAMA therapy, no significant difference in re-hospitalization or death was observed between the two groups (Figure 2C).

\section{Discussion}

This study showed a reduced risk of re-hospitalization for COPD exacerbation or death in the Dai-kenchu-to group vs control group among $\geq 75$-year-old patients with COPD, using 1-to-4 propensity score matching. The subgroup analysis of patients without LAMA therapy showed no significant difference in the outcome between the Dai-kenchu-to users and non-users. Our findings indicate that Dai-kenchu-to may reduce the risk of severe COPD exacerbation requiring hospitalization, particularly in advanced-age patients with COPD, possibly by improving treatment adherence in LAMA users. Our study is the first to evaluate the relationship between Dai-kenchu-to (TU-100) and COPD. We also attempted to provide a bridge between the concept of traditional Oriental herbal medicine and modern medicine.

We were not able to clearly show the mechanisms that explain our findings in the present study. Despite numerous studies demonstrating the effect and mechanism ${ }^{9-17}$ of Dai-kenchu-to in the gut, no studies have yet evaluated the additional benefits of Dai-kenchu-to in the organs outside the digestive system. However, there are several possible explanations for why Dai-kenchu-to was preferable in advancedage patients with COPD, especially in LAMA users. Improved gut motility is a major effect of Dai-kenchu-to, and this can be a plausible mechanism for reducing exacerbation in advanced-age patients with COPD. In general, patients of advanced age are susceptible to abdominal bloating and/or constipation. ${ }^{19-21}$ Patients aged $>75$ years using LAMAs are likely to have an advanced stage of COPD with a higher risk of frailty and reduced gastrointestinal motility than those not using LAMAs, which may explain their response to the treatment with Dai-kenchu-to. Moreover, such patients are vulnerable to adverse drug reactions, ${ }^{22}$ which may also be true of LAMAs. Dai-kenchu-to may show improved patient adherence to long-acting bronchodilators, particularly advanced-age patients with COPD experiencing abdominal bloating and/or constipation, resulting in a lower risk of re-hospitalization.

Furthermore, because the thoracic lumen shares the diaphragm with the abdominal lumen, the diaphragmatic response to abdominal loading inevitably affects the lung function, particularly in advanced-age patients with COPD whose costal wall is unlikely to further expand horizontally to compensate for the limitation in vertical extension of the lung. ${ }^{23}$ In fact, diaphragmatic motion during tidal breathing is reportedly greater in patients with than without COPD, suggesting a pivotal role of diaphragmatic motion in compensation for breathlessness. ${ }^{24}$ Abdominal bloating and/or constipation may interfere with diaphragmatic motion and may aggravate symptoms in advanced-age patients with COPD, resulting in reduced physical activity and thereby increasing the risk of COPD exacerbation. ${ }^{25}$ Further studies are required to assess whether the effect of Dai-kenchu-to simply relies on the improvement of gut motility.

Intestinal hyperpermeability following splanchnic hypoxia, possibly caused by blood flow redistribution, may lead to increased bacterial translocation in patients with COPD. ${ }^{7,8}$ Dai-kenchu-to in both animals ${ }^{26}$ and humans ${ }^{27}$ increases intestinal blood flow. A protective effect of Daikenchu-to against bacterial translocation has been shown in rats. ${ }^{28-30}$ Dai-kenchu-to may have reduced the risk of COPD exacerbation in older patients in the present study by preserving intestinal blood flow and preventing bacterial translocation.

In addition, disruption of the microbiome in patients with lung disease, including COPD, has been reviewed, ${ }^{31}$ and the modulatory effect of Dai-kenchu-to on the microbiome has been studied. ${ }^{32,33}$ In one study, Dai-kenchu-to preserved the diversity of the microbiome in stressed mice. In another study, it reshaped the proportion of the microbiome with increased short-chain fatty acids in rats. The increased short-chain fatty acids reportedly suppressed inflammation in the lungs and were protective in a mouse model of elastase-induced emphysema. ${ }^{34}$ Moreover, short-chain fatty acids have been 

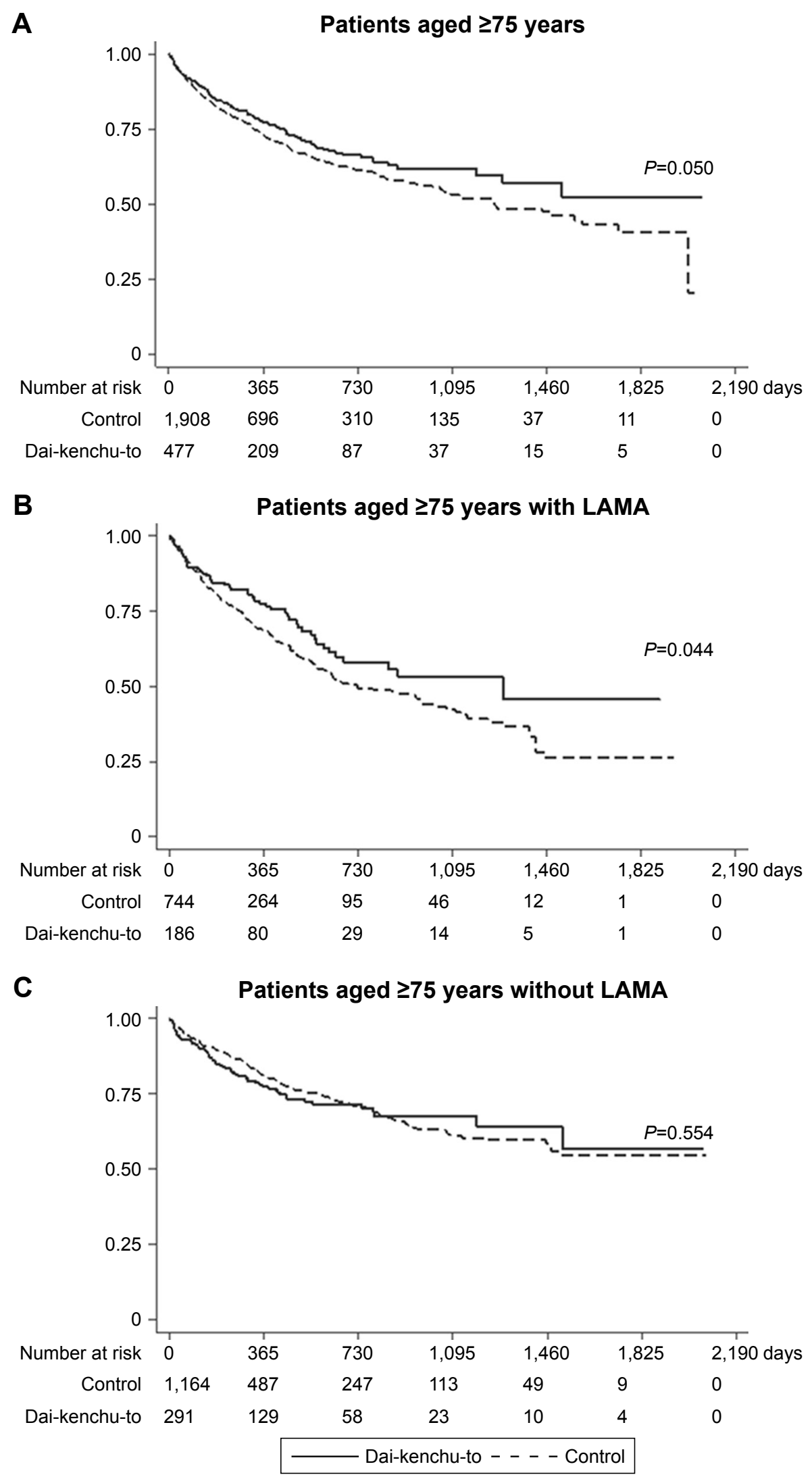

Figure 2 Kaplan-Meier survival curves of patients with COPD with or without Dai-kenchu-to after hospitalization for COPD exacerbation in the I-to-4 propensity score-matched population. (A) Results for patients aged $\geq 75$ years. (B) Results for patients with LAMA therapy. (C) Results for patients without LAMA therapy before hospitalization. The Kaplan-Meier curves were compared with a log-rank test. A $P$-value of $<0.05$ indicated statistical significance. Abbreviation: LAMA, long-acting muscarinic receptor antagonist. 
Table 3 HRs of re-hospitalization for COPD exacerbation or death after I-to-4 propensity score matching in the Dai-kenchu-to group vs control group

\begin{tabular}{l|l|l|l|l|l}
\hline Patients & \multicolumn{2}{l|}{ Number of patients } & HR & 95\% CI & P-value \\
\cline { 2 - 3 } & Control & Dai-kenchu-to & & & \\
\hline All patients & $\mathrm{I}, 908$ & 477 & 0.82 & $0.67-0.99$ & 0.050 \\
Subgroup analyses & & & 0.73 & $0.54-0.99$ & 0.045 \\
$\quad$ LAMA users & 744 & 186 & 1.09 & $0.83-1.43$ & 0.555 \\
LAMA non-users & $\mathrm{I}, 164$ & 291 & & \\
\hline
\end{tabular}

Abbreviation: LAMA, long-acting muscarinic receptor antagonist.

implicated in the immune response $\mathrm{e}^{35}$ and non-eosinophilic hyperresponsiveness in the airway. ${ }^{36}$ The modulatory effect of Dai-kenchu-to on the microbiome in the gut may serve as a basis for further exploration of the mechanism of Daikenchu-to in advanced-age patients with COPD.

The capability of Dai-kenchu-to to activate transient receptor potential ankyrin (TRPA) channels or transient receptor potential vanilloid (TRPV) channels is also of interest. ${ }^{11}$ Activation of TRPA and TRPV channels on vagal sensory nerves in the airways initiates the cough reflex, which is considered to be a therapeutic target when excessive in disease states. ${ }^{37}$ The protective effect of cough (ie, clearing harmful particles from the airway) is also acknowledged, particularly in stable states. However, whether Dai-kenchu-to can activate TRPA or TRPV channels in the airway has not been demonstrated. Therefore, further in vivo studies are required.

Several limitations of this study should be acknowledged. First, patients who switched hospitals during the study period and patients whose outpatient care hospital differed from the hospital for hospitalization were not captured. This may be a potential source of confounding. Second, the database does not contain data for each patient's pulmonary function tests and symptoms. We, therefore, evaluated medications for COPD in the stable states (before hospitalization) as a surrogate for pulmonary function tests and symptoms. Third, factors such as the current smoking status and actual baseline physical activity, both of which may affect the outcomes, were not recorded in the DPC database. Fourth, in our propensity score-matching analysis, some variables such as residential regions, Hugh-Jones dyspnea, and activities of daily living at discharge were not well balanced. However, we performed a reanalysis with these three variables included as independent variables in the Cox regression model after 1-to-4 propensity score matching and obtained a similar result (HR, 0.81; 95\% CI, 0.66-0.99; $P=0.039$ ).

\section{Conclusion}

Our study suggests that Dai-kenchu-to may reduce the risk of re-hospitalization for COPD exacerbation, most likely by improving the adherence to LAMAs in advanced-age patients with COPD. Prospective studies to confirm our findings and to elucidate the mechanisms of the protective effect of Dai-kenchu-to in advanced-age patients with COPD are needed.

\section{Acknowledgments}

This work was supported by grants from the Ministry of Health, Labor and Welfare, Japan (H30-Policy-Designated-001, H30-Policy-Designated-004, and H29-ICTGenral-004) and the Ministry of Education, Culture, Sports, Science and Technology, Japan (17H04141). The funding bodies had no role in the design of the study; collection, analysis, or interpretation of the data; or writing of the manuscript.

\section{Author contributions}

TJ: study design, data analysis, data interpretation, and manuscript preparation. NM: data analysis, data interpretation, and manuscript preparation. HY: data analysis, data interpretation, and manuscript preparation. YS: data analysis and data interpretation. HM: data collection, data analysis, and data interpretation. HU: study design and data interpretation. AM: study design and data interpretation. YY: study design and data interpretation. KF: data collection and data interpretation. HY: study design and data interpretation. YH: study design, data interpretation, and manuscript preparation. All authors contributed to data analysis, draft or revising the article, gave final approval of the version to be published, and agree to be accountable for all aspects of the work.

\section{Disclosure}

TJ (corresponding author), NM, and HY have received financial contributions from Tsumura \& Company (Tokyo, Japan) because they have academic affiliations with the Department of Health Services Research, Graduate School of Medicine, The University of Tokyo supported by Tsumura \& Company. Tsumura \& Company played no role in this study. The corresponding author also receives research funding from Tsumura \& Company for research not related to this study. The authors report no other conflicts of interest in this work. 


\section{References}

1. Jones PW, Watz H, Wouters EF, Cazzola M. COPD: the patient perspective. Int J Chron Obstruct Pulmon Dis. 2016;11(Spec Iss):13-20.

2. George SE, Borello-France DF. Perspective on physical therapist management of functional constipation. Phys Ther. 2017;97(4):478-493.

3. McGarvey L, Lee AJ, Roberts J, Gruffydd-Jones K, McKnight E, Haughney J. Characterisation of the frequent exacerbator phenotype in COPD patients in a large UK primary care population. Respir Med. 2015;109(2):228-237.

4. GOLD reports 2018: Global strategy for the diagnosis, management, and prevention of chronic obstructive pulmonary disease. Available from: https://goldcopd.org/wp-content/uploads/2017/11/GOLD-2018-v6.0FINAL-revised-20-Nov_WMS.pdf. Accessed December 19, 2018.

5. Rabe KF, Watz H. Chronic obstructive pulmonary disease. Lancet. 2017;389(10082):1931-1940.

6. Hanlon P, Nicholl BI, Jani BD, et al. Examining patterns of multimorbidity, polypharmacy and risk of adverse drug reactions in chronic obstructive pulmonary disease: a cross-sectional UK Biobank study. BMJ Open. 2018;8(1):e018404.

7. Rutten EPA, Lenaerts K, Buurman WA, Wouters EFM. Disturbed intestinal integrity in patients with COPD: effects of activities of daily living. Chest. 2014;145(2):245-252.

8. Sprooten RTM, Lenaerts K, Braeken DCW, et al. Increased small intestinal permeability during severe acute exacerbations of COPD. Respiration. 2018;95(5):334-342.

9. Akamaru Y, Takahashi T, Nishida T, et al. Effects of daikenchuto, a Japanese herb, on intestinal motility after total gastrectomy: a prospective randomized trial. J Gastrointest Surg. 2015;19(3):467-472.

10. Kawasaki N, Nakada K, Suzuki Y, Furukawa Y, Hanyu N, Kashiwagi H. Effect of Dai-kenchu-to on gastrointestinal motility and gastric emptying. Int J Surg. 2009;7(3):218-222.

11. Kono T, Shimada M, Yamamoto M, et al. Complementary and synergistic therapeutic effects of compounds found in Kampo medicine: analysis of Daikenchuto. Front Pharmacol. 2015;6(73):159.

12. Yasunaga H, Miyata H, Horiguchi H, Kuwabara K, Hashimoto H, Matsuda S. Effect of the Japanese herbal Kampo medicine Daikenchu-to on postoperative adhesive small bowel obstruction requiring long-tube decompression: a propensity score analysis. Evid Based Complement Alternat Med. 2011;2011:264289.

13. Ishizuka M, Shibuya N, Nagata H, et al. Perioperative administration of traditional Japanese herbal medicine Daikenchuto relieves postoperative ileus in patients undergoing surgery for gastrointestinal cancer: a systematic review and meta-analysis. Anticancer Res. 2017;37(11): 5967-5974.

14. Katsuno H, Maeda K, Ohya M, et al. Clinical pharmacology of Daikenchuto assessed by transit analysis using radiopaque markers in patients with colon cancer undergoing open surgery: a multicenter double-blind randomized placebo-controlled study (JFMC39-0902 additional study). J Gastroenterol. 2016;51(3):222-229.

15. Kaido T, Shimamura T, Sugawara Y, et al. Multicentre, randomised, placebo-controlled trial of extract of Japanese herbal medicine Daikenchuto to prevent bowel dysfunction after adult liver transplantation (DKB 14 Study). BMJ Open. 2015;5(9):e008356.

16. Tsuda H, Kotani T, Sumigama S, Mano Y, Hayakawa H, Kikkawa F. Efficacy and safety of Daikenchuto (TJ-100) in pregnant women with constipation. Taiwan J Obstet Gynecol. 2016;55(1):26-29.

17. Yuki M, Komazawa Y, Kobayashi Y, et al. Effects of Daikenchuto on abdominal bloating accompanied by chronic constipation: a prospective, single-center randomized open trial. Curr Ther Res Clin Exp. 2015;77: $58-62$.
18. Rosenbaum PR, Rubin DB. Constructing a control group using multivariate matched sampling methods that incorporate the propensity score. Am Stat. 1985;39:33-38.

19. Fávaro-Moreira NC, Krausch-Hofmann S, Matthys C, et al. Risk factors for malnutrition in older adults: a systematic review of the literature based on longitudinal data. Adv Nutr. 2016;7(3):507-522.

20. K Bailes, Reeve K. Constipation in older adults. Nurse Pract. 2013;3838: $21-25$

21. Gau JT, Acharya UH, Khan MS, Kao TC. Risk factors associated with lower defecation frequency in hospitalized older adults: a case control study. BMC Geriatr. 2015;15(1):44.

22. Wooten JM. Adverse drug reactions: part I. South Med J. 2010;103: $1025-1028$.

23. Barba E, Quiroga S, Accarino A, et al. Mechanisms of abdominal distension in severe intestinal dysmotility: abdomino-thoracic response to gut retention. Neurogastroenterol Motil. 2013;25(6):e389-e394.

24. Yamada Y, Ueyama M, Abe T, et al. Difference in diaphragmatic motion during tidal breathing in a standing position between COPD patients and normal subjects: time-resolved quantitative evaluation using dynamic chest radiography with flat panel detector system ("dynamic X-ray phrenicography"). Eur J Radiol. 2017;87:76-82.

25. Katajisto M, Koskela J, Lindqvist A, Kilpeläinen M, Laitinen T. Physical activity in COPD patients decreases short-acting bronchodilator use and the number of exacerbations. Respir Med. 2015;109(10):1320-1325.

26. Kono T, Omiya Y, Hira Y, et al. Daikenchuto (TU-100) ameliorates colon microvascular dysfunction via endogenous adrenomedullin in Crohn's disease rat model. J Gastroenterol. 2011;4646(1):1187-1196.

27. Takayama S, Seki T, Watanabe M, et al. The herbal medicine Daikenchuto increases blood flow in the superior mesenteric artery. Tohoku J Exp Med. 2009;219(4):319-330.

28. Takasu C, Yismaw WG, Kurita N, et al. TU-100 exerts a protective effect against bacterial translocation by maintaining the tight junction. Surg Today. 2017;47(10):1287-1294.

29. Yada K, Ishibashi H, Mori H, et al. The Kampo medicine "Daikenchuto (TU-100)" prevents bacterial translocation and hepatic fibrosis in a rat model of biliary atresia. Surgery. 2016;159(6):1600-1611.

30. Yoshikawa K, Kurita N, Higashijima J, et al. Kampo medicine "Daikenchu-to" prevents bacterial translocation in rats. Dig Dis Sci. 2008; 53(7):1824-1831.

31. Shukla SD, Budden KF, Neal R, Hansbro PM. Microbiome effects on immunity, health and disease in the lung. Clin Transl Immunology. 2017;6(3):e133

32. Yoshikawa K, Shimada M, Kuwahara T, et al. Effect of Kampo medicine "Dai-kenchu-to" on microbiome in the intestine of the rats with fast stress. J Med Invest. 2013;60(3-4):221-227.

33. Hasebe T, Ueno N, Musch MW, et al. Daikenchuto (TU-100) shapes gut microbiota architecture and increases the production of ginsenoside metabolite compound K. Pharmacol Res Perspect. 2016;4(1): $\mathrm{e} 00215$.

34. Tomoda K, Kubo K, Dairiki K, et al. Whey peptide-based enteral diet attenuated elastase-induced emphysema with increase in short chain fatty acids in mice. BMC Pulm Med. 2015;15(1):64.

35. Trompette A, Gollwitzer ES, Yadava K, et al. Gut microbiota metabolism of dietary fiber influences allergic airway disease and hematopoiesis. Nat Med. 2014;20(2):159-166.

36. Cho Y, Abu-Ali G, Tashiro H. The microbiome regulates pulmonary responses to ozone in mice. Am J Respir Cell Mol Biol. 2018; 59(3):346-354.

37. Bonvini SJ, Birrell MA, Smith JA, Belvisi MG. Targeting TRP channels for chronic cough: from bench to bedside. Naunyn Schmiedebergs Arch Pharmacol. 2015;388(4):401-420. 


\section{Supplementary materials}

Table SI List of drugs used to treat COPD and drugs used to define COPD exacerbation in outpatient settings

\begin{tabular}{|l|l|}
\hline \multicolumn{2}{|l|}{ Medications for COPD } \\
\hline ICS & Beclomethasone, fluticasone, budesonide, ciclesonide, mometasone \\
\hline LAMA & Titropium, glycopyrronium, umeclidinium \\
\hline LABA & Salmeterol, formoterol, indacaterol, vilanterol, tulobuterol \\
\hline $\begin{array}{l}\text { Other medications for } \\
\text { COPD }\end{array}$ & $\begin{array}{l}\text { Theophylline, acetylcysteine, ambroxol, carbocisteine, fudosteine, bromhexine, salbutamol, fenoterol, procaterol, } \\
\text { ipratropium, oxitropium }\end{array}$ \\
\hline Drugs used for COPD exacerbation \\
\hline Systemic corticosteroids & Dexamethasone, paramethasone, prednisolone, methylprednisolone, betamethasone, triamcinolone, hydrocortisone \\
\hline Antibiotics & $\begin{array}{l}\text { Benzylpenicillin, aspoxicillin, amoxicillin, ampicillin, amoxicillin/clavulanate, bacampicilin, cinoxacin piperacillin, } \\
\text { pivemecillinam, sulbactam/ampicillin, tazobactam/piperacillin, abpc-mcipc, cefroxadine, cefaclor, cefalex, cefalotin, } \\
\text { cefazolin, cefuroxime, cefotiam, cefmetazol, cefotiam, flomoxef, cefminox, cefodizime, latamoxef, sulbactam/ } \\
\text { cefoperazone, cefotaxime, cefoperazone, cefixime, ceftibuten, cefbuperazone, cefcapene pivoxil, cefteram pivoxil, } \\
\text { cefpodoxime proxetil, cefteram pivoxil cefpirome, cefmenoxime, csfditoren, ceftazidime, ceftriaxone, cefozopran, } \\
\text { cefepime, ceftizoxime, telithromycin, clindamycin, amikacin isepamicin, gentamicin, tobramycin, didekacin, aztreonam, } \\
\text { erythromycin, clarithromycin, azithromycin, josamycin, roxithromycin, aztreonam, tosufloxacin, ofloxacin, garenoxacin, } \\
\text { levofloxacin, sitafloxacin, ciprofloxacin, tosufloxacin, norfloxacin, pazufloxacin, prulifloxacin, moxifloxacin, lomefloxcin, } \\
\text { faropenem, biapenem, tebipenem pivoxil, panipenem/betamipron, imipenem/cilastatin, doripenem, meropenem }\end{array}$ \\
\hline Anti-flu medicine & \begin{tabular}{l} 
Oseltamivir, zanamivir, peramivir, laninamivir \\
\hline
\end{tabular}
\end{tabular}

Abbreviations: ICS, inhaled corticosteroids; LABA, long-acting $\beta$-agonist; LAMA, long-acting muscarinic receptor antagonist.

Table S2 ICD- 10 codes used to identify comorbidities

\begin{tabular}{|c|c|}
\hline Comorbidity & ICD- 10 codes \\
\hline Lung cancer & $\mathrm{C} 34$ \\
\hline Other malignancy & C00-26, C30-33, C37-4I, C43-58, C60-76 \\
\hline Interstitial pneumonia & B22I, J70I, J704, J84I, J848, J849, J990, J99I, M32I, M330, M33I, M332, M35I \\
\hline Bronchial asthma & J45, J46 \\
\hline Bronchiectasis & $\mathrm{J} 40, \mathrm{~J} 4 \mathrm{I}, \mathrm{J} 42, \mathrm{~J} 47$ \\
\hline Bacterial lower respiratory tract infection & A48I, J100, JII0, JI3-I6, JI70, JI78, J18, J85, J86 \\
\hline Mycobacterium infection & Al50-154, Al56-159, Al6I-162, Al65, Al68-169, A19, A310, A319 \\
\hline Mycotic infection & A420, A43, B37, B380-382, B390-392, B400-402, B4I0, B420, B440, B44I, B449, B460, JI72 \\
\hline Cor pulmonale & 127 \\
\hline Congestive heart failure & $E 059,146,150,1099,1110$ \\
\hline Ischemic heart disease & $120-25$ \\
\hline Tachycardia & 147-49, R000, T818 \\
\hline Autoimmune disease & M05, M06, M08, M30-35 \\
\hline Stroke & $160-64$ \\
\hline Liver dysfunction & B89, BI8I, BI82, B659, B66I, K702, K703, K72, K74, K76I, K762, K763, K766, K767 \\
\hline Renal failure & EI02, EII2, EI42, II20, NI7-19 \\
\hline GERD & $\mathrm{K} 2 \mathrm{I}$ \\
\hline Constipation and/or ileus & K56, K590 \\
\hline Prostate hypertrophy & N40 \\
\hline
\end{tabular}

Abbreviation: GERD, gastroesophageal reflux disease. 
International Journal of COPD

\section{Publish your work in this journal}

The International Journal of COPD is an international, peer-reviewed journal of therapeutics and pharmacology focusing on concise rapid reporting of clinical studies and reviews in COPD. Special focus is given to the pathophysiological processes underlying the disease, intervention programs, patient focused education, and self management protocols.

This journal is indexed on PubMed Central, MedLine and CAS. The manuscript management system is completely online and includes a very quick and fair peer-review system, which is all easy to use. Visit http://www.dovepress.com/testimonials.php to read real quotes from published authors 